\title{
Large- and Small-Signal IMD Behavior of Microwave Power Amplifiers
}

\author{
Nuno Borges de Carvalho, Student Member, IEEE, and José Carlos Pedro, Member, IEEE
}

\begin{abstract}
In this paper, large-signal intermodulation distortion (IMD) sweet spots in microwave power amplifiers are studied and predicted using a new mathematical basis. The variations in the IMD versus drive pattern with active bias point and the terminating matching networks are investigated. This nonlinear distortion model enabled the design of power amplifiers specially tailored to present a desired IMD versus drive-level pattern. For practical validation purposes, a MESFET case study and an illustrative application example will be presented.
\end{abstract}

Index Terms-Describing function, intermodulation distortion, power amplifiers, Volterra series.

\section{INTRODUCTION}

$\mathbf{O}$ NE OF THE major issues in a power-amplifier design process is the level of nonlinear distortion allowed, in order to fulfil the specifications of SNR.

Normally, to solve this problem, the input backoff technique is used, which is also known for its efficiency degradation and, thus, on the overall telecommunications' system performance.

The main objective of this paper is to present a new and rigorously founded mathematical model for the nonlinear distortion mechanisms responsible for the so-called largesignal intermodulation distortion (IMD) sweet spots in power amplifiers [1]. That enabled the explanation of not quite understood and, thus, sometimes referred to as "strange," behaviors of the most important distortion effects, e.g., inband IMD, spectral regrowth, and harmonic distortion, which could then be used to overcome some of the associated input backoff problems.

Usual power-amplifier's fundamental output power and inband IMD curves versus input power can present two clearly distinct behaviors, as is shown in Fig. 1.

The continuous curve in Fig. 1 corresponds to a normal gain compression operation, in which output power first follows linearly the input and then, for a determined input drive, progressively compresses. Beyond this point, the relation between input and output is less than $1 \mathrm{~dB} / \mathrm{dB}$, and tends to an asymptotic constant. The corresponding in-band distortion begins with a cubic behavior, rising $3 \mathrm{~dB}$ for each decibel of

Manuscript received March 26, 1999; revised July 9, 1999. This work was supported in part by the Portuguese Science Bureau, Fundação para a Ciência e Tecnologia, under Project 2/2.1/TIT1562/95-ISAC and under Project PBIC/TIT/1766/93-ITCOM.

The authors are with the Instituto de Telecomunicações, Universidade de Aveiro, 3810 Aveiro, Portugal.

Publisher Item Identifier S 0018-9480(99)08432-X.

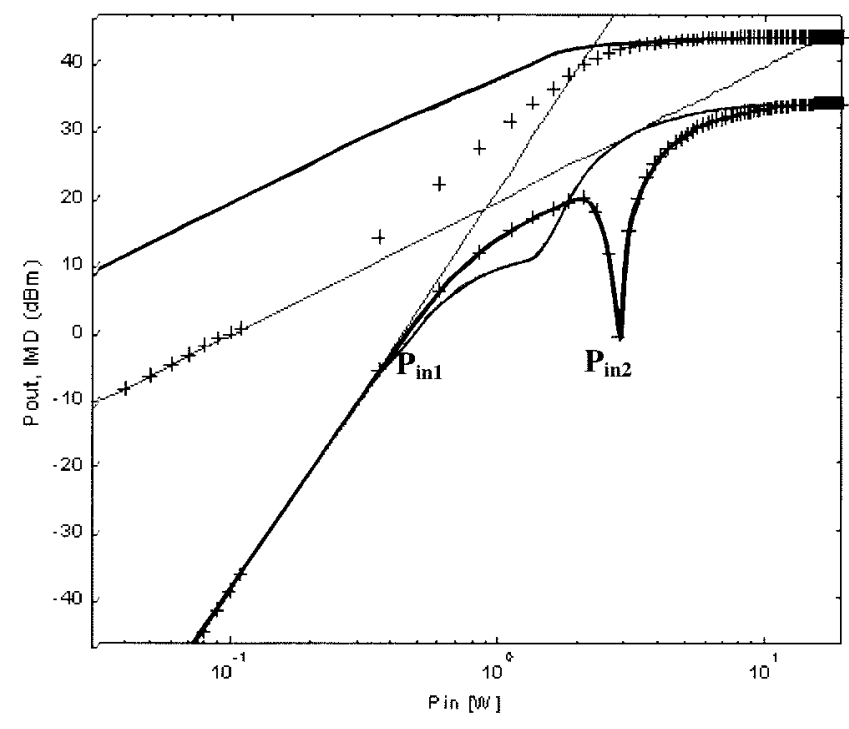

Fig. 1. $P_{\text {in }}-P_{\text {out }}$ and IMD usual curves.

input power. Also, when the fundamental starts to compress, it presents a sudden increase, tending again to a constant. Thus, the output signal power-to-distortion ratio $(\mathrm{C} / \mathrm{I})$ rapidly degrades.

On the other hand, the curve of Fig. 1, marked with (+), represents the case of sometimes observed gain expansion phenomena. That means the output signal power first linearly follows the input power, then it experiences a faster rate of rise (gain expansion), and finally, it compresses again to the above maximum output power.

Now, consider the correspondent in-band IMD curve. First, it follows the previous curve, within the small-signal regime. Contrary to the previous case, it then presents an unexpected minimum (large-signal IMD sweet spot) at a certain input power. By observing the two curves, and remembering that the design goal is a prescribed $(\mathrm{C} / \mathrm{I})$, it is no longer evident which input backoff should be considered for this latter design. In fact, for the same distortion characteristics, we will get a better output $\mathrm{C} / \mathrm{I}$ by selecting $\mathbf{P}_{\mathrm{in} 2}$ as the amplifier working point than with $\mathbf{P}_{\text {in1 }}$.

Other authors [1], [2] have already observed this kind of behavior in IMD. In [1], it is presented for bipolar junction transistor (BJT) and lateral diffused MOSFET (LDMOS) power amplifiers. In [2], the author not only observes it, as he tries to approximate it using a polynomial model. 


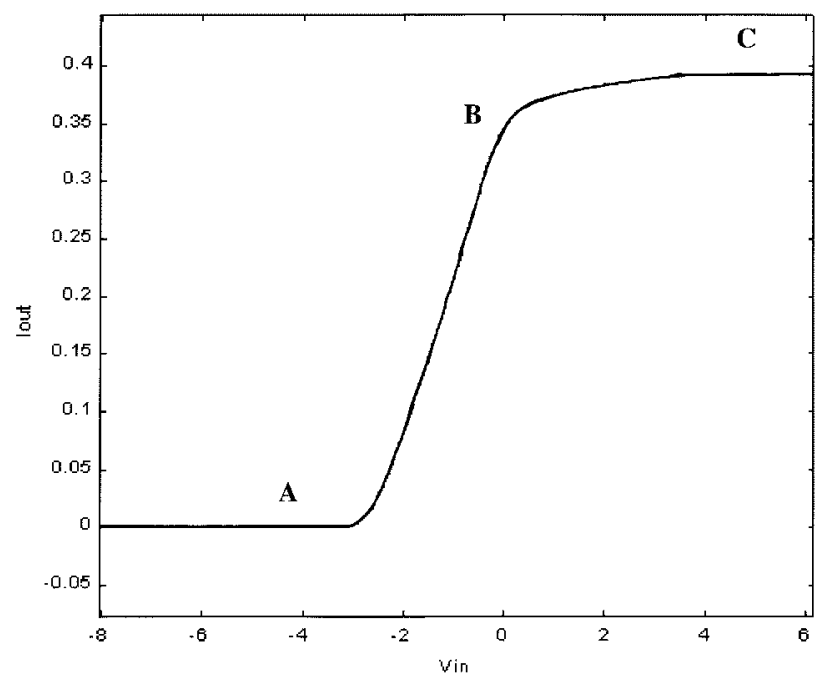

Fig. 2. Characteristic function.

However, as far as we know, until now, no one tried to relate its characteristics to the active device's nonlinearities or even predict it without having to measure the final amplifier circuit.

Since the problem resides on how to predict these different amplifier behaviors, we began by analyzing the amplifier in its small-signal regime, and then extended the results to large-signal operation. Small-signal calculations were based on a standard Volterra series (VS) analysis [3] (VS expansion of the amplifier's active device nonlinear model $\left.-I_{\text {out }}\left(V_{\text {in }}\right)\right)$. However, due to the well-known limited convergence radius provided by this method, the large signal was to be handled by describing function techniques [4]. From the integration of these small- and large-signal amplifier modeling methods, a new behavioral distortion model was then proposed.

In the following sections, this novel amplifier model is presented and applied to a simple MESFET amplifier circuit. Finally, it is validated by showing some application examples where the transistor's quiescent point (for Class-B and Class$\mathrm{AB}$ operation) and the input stimuli (two-tone and narrowband Gaussian noise) were varied.

\section{MathematicAl APPROACH}

Consider a nonlinear device having a characteristic function similar to the one presented in Fig. 2, where the $x x$-axis is some input control variable, like voltage, and the $y y$-axis is the output variable, as current. The study of this nonlinear function will be divided into two sub-analyses.

As indicated in Section I, small-signal analysis will be derived from the VS expansion of that nonlinear transfer characteristic $\left[I_{\text {out }}=f_{N L}\left(V_{\mathrm{in}}\right)\right]$ around some preselected quiescent point (in this case, a simple Taylor expansion since the transfer function is memoryless).

In that sense, a good approximation of the characteristic function between $\mathbf{A}$ and $\mathbf{B}$ can be expected with only a fifthdegree Taylor series, provided the expansion point is also within that range. We called that the small-signal regime, from which output current can be given by

$$
\begin{aligned}
i_{\text {out }}(t)= & \frac{1}{2} H_{1} \sum_{q=-Q}^{Q} V_{1 q} e^{j \omega_{q} t} \\
& +\frac{1}{4} H_{2} \sum_{q_{1}=-Q}^{Q} \sum_{q_{2}=-Q}^{Q} V_{1 q_{1}} V_{1 q_{2}} e^{j\left(\omega_{q_{1}}+\omega_{q_{2}}\right) t} \\
& +\frac{1}{8} H_{3} \sum_{q_{1}=-Q}^{Q} \sum_{q_{2}=-Q}^{Q} \\
& \cdot \sum_{q_{3}=-Q}^{Q} V_{1 q_{1}} V_{1 q_{2}} V_{1 q_{3}} e^{j\left(\omega_{q_{1}}+\omega_{q_{2}}+\omega_{q_{3}}\right) t} \\
& +\frac{1}{16} H_{4} \sum_{q_{1}=-Q}^{Q} \sum_{q_{2}=-Q}^{Q} \sum_{q_{3}=-Q}^{Q} \\
& \cdot \sum_{q_{4}=-Q}^{Q} V_{1 q_{1}} V_{1 q_{2}} V_{1 q_{3}} V_{1 q_{4}} e^{j\left(\omega_{q_{1}}+\omega_{q_{2}}+\omega_{q_{3}}+\omega_{q_{4}}\right) t} \\
& +\frac{1}{32} H_{\tilde{5}} \sum_{q_{1}=-Q}^{Q} \sum_{q_{2}=-Q}^{Q} \sum_{q_{3}=-Q}^{Q} \sum_{q_{4}=-Q}^{Q} \\
& \cdot \sum_{q_{5}=-Q}^{Q} V_{1 q_{1}} V_{1 q_{2}} V_{1 q_{3}} V_{1 q_{4}} V_{1 q_{5}} \\
& \cdot e^{\left.j\left(\omega_{q_{1}}+\omega_{q_{2}}+\omega_{q_{3}}+\omega_{q_{4}}\right)+\omega_{q_{5}}\right) t} \ldots .
\end{aligned}
$$

If the input is assumed as a sinusoidal two-tone

$$
\begin{aligned}
v_{\text {in }}(t)=A & {\left[\cos \left(\omega_{1} t+\phi_{1}\right)+\cos \left(\omega_{2} t+\phi_{2}\right)\right] } \\
=\frac{A}{2}\left[e^{j\left(\omega_{1} t+\phi_{1}\right)}+e^{-j\left(\omega_{1} t+\phi_{1}\right)}+e^{j\left(\omega_{2} t+\phi_{2}\right)}\right. & \\
& \left.+e^{-j\left(\omega_{2} t+\phi_{2}\right)}\right]
\end{aligned}
$$

then the in-band IMD behavior at frequency $2 \omega_{2}-\omega_{1}$ will be

$$
\begin{aligned}
\mathrm{SS}_{I_{\text {out }}} & \left(2 \omega_{2}-\omega_{1}\right) \\
= & \frac{3 A^{2}}{8} H_{3}\left(\omega_{2}, \omega_{2},-\omega_{1}\right) e^{j\left[\left(2 \omega_{2}-\omega_{1}\right) t+2 \phi_{2}-\phi_{1}\right]} \\
& +\frac{50 A^{5}}{32} H_{5}\left(\omega_{2}, \omega_{2},-\omega_{1}, \omega,-\omega\right) \\
& \cdot e^{j\left[\left(2 \omega_{2}-\omega_{1}\right) t+2 \phi_{2}+\phi_{1}\right]}
\end{aligned}
$$

where SS stands for small-signal part of the response.

In order to have an IMD minimum, we must have

$$
\begin{aligned}
& \frac{3 A^{3}}{8} H_{3}\left(\omega_{2}, \omega_{2},-\omega_{1}\right) e^{\left[\left(2 \omega_{2}-\omega_{1}\right) t+2 \phi_{2}-\phi_{1}\right]} \\
&=- \frac{50 A^{2}}{32} H_{5}\left(\omega_{2}, \omega_{2},-\omega_{1}, \omega,-\omega\right) \\
& \cdot e^{j\left[\left(2 \omega_{2}-\omega_{1}\right) t+2 \phi_{2}-\phi_{1}\right]} .
\end{aligned}
$$

Accordingly, if $H_{5}(\cdot)$ has an opposite phase to $H_{3}(\cdot)$, the minimum will appear at an input power of $P_{\text {in }}=$ $\left|4 H_{3}(\cdot) / 25 H_{5}(\cdot)\right|$.

If a larger input signal is now considered, like one that travels far beyond B, a higher Taylor series degree and, thus, $H_{i}(\cdot)$ must be used in order to maintain VS' accuracy. Thus, 


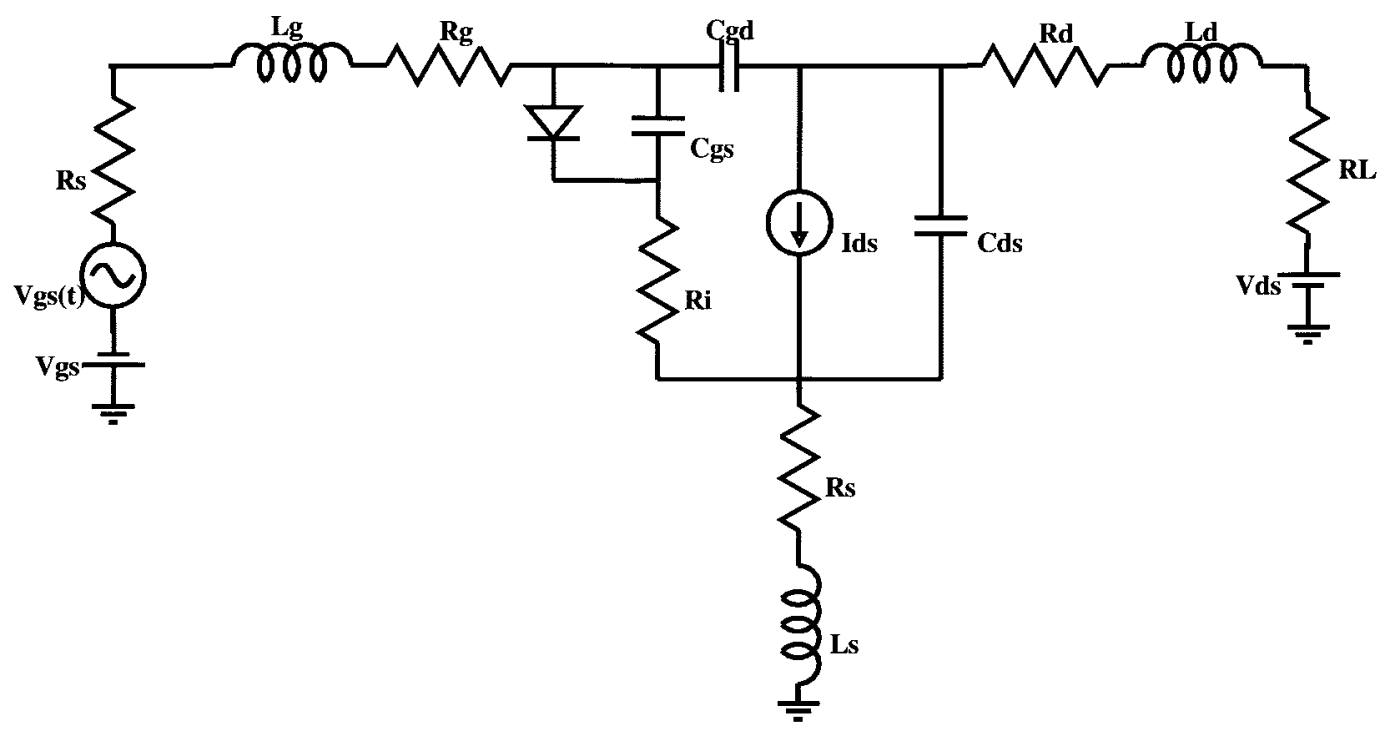

Fig. 3. Schematic diagram of the power-amplifier circuit.

by combining different arrangements of $H_{i}(\cdot)$, various IMD power nulls may be predicted. It can be shown that usual microwave amplifier's behavior can be very well represented until point $\mathbf{B}$ using a simple fifth or seventh VS approximation, although many more terms should be used for a signal that travels up to $\mathbf{C}$. This makes the applicability of the VS inadequate for large signal, asking for another technique like the two sinusoidal input describing function (TSIDF) [4].

The derivation of the TSIDF for the transfer characteristic of Fig. 2 demands for a two-tone input excitation, which leads to

$$
\begin{aligned}
I_{\text {out }} & \left(A, n_{1} \omega_{1}, n_{2} \omega_{2}\right) \\
= & \frac{2 j}{A T_{1} T_{2}} \int_{-T_{1} / 2}^{T_{1} / 2} \int_{-T_{2} / 2}^{T_{2} / 2} f_{N L}\left[v_{\text {in }}(t)\right] \\
& \cdot e^{-j n_{1} \omega_{1} t_{1}} e^{-j n_{2} \omega_{2} t_{2}} d t_{1} d t_{2} \\
= & \frac{2 j}{A T_{1} T_{2}} \int_{-T_{1} / 2}^{T_{1} / 2} \int_{-T_{2} / 2}^{T_{2} / 2} f_{N L}\left[A \sin \left(\omega_{1} t\right)+A \sin \left(\omega_{2} t\right)\right] \\
& \cdot e^{-j n_{1} \omega_{1} t_{1}} e^{-j n_{2} \omega_{2} t_{2}} d t_{1} d t_{2}
\end{aligned}
$$

where $v_{\text {in }}(t)=A \sin \left(\omega_{1} t\right)+A \sin \left(\omega_{2} t\right)$. Then,

$$
\begin{aligned}
& \operatorname{TS}_{\operatorname{Lif}}\left(A, 2 \omega_{2}-\omega_{1}\right) \\
& =\frac{2 j}{A T_{1} T_{2}} \int_{-T_{1} / 2}^{T_{1} / 2} \int_{-T_{2} / 2}^{T_{2} / 2} f_{N L}\left[A \sin \left(\omega_{1} t_{1}\right)+A \sin \left(\omega_{2} t_{2}\right)\right] \\
& \quad \cdot e^{j \omega_{1} t_{1}} e^{-j 2 \omega_{2} t_{2}} d t_{1} d t_{2} .
\end{aligned}
$$

Using these expressions, and taking into account the saturation shape of $\left[I_{\text {out }}=f_{N L}\left(V_{\text {in }}\right)\right]$ represented in Fig. 2, it can be shown that the IMD component at $2 \omega_{2}-\omega_{1}$ converges to a precisely determined constant power with $180^{\circ}$ out of phase (compared with the fundamental output power), when the input excursion tends to infinity (large-signal regime). Thus, while the VS accurately describes the amplifier IMD at small signal, the describing function technique is the appropriate choice for large-signal regimes.

In conclusion, small- and large-signal distortion regimes can be easily integrated into the same mathematical formulation.
Section III will be devoted to the presentation and discussion of such a new distortion model.

\section{SMALL- AND LARGE-Signal BeHAVIOR ModelS}

The integration of small- and large-signal distortion led to the simultaneous use of a Volterra model associated with a describing function one. Thus, the mathematical formulation is

$$
\begin{aligned}
I_{\text {out }}\left(2 \omega_{2}-\omega_{1}\right)= & \frac{3 A^{3}}{8} H_{3}\left(\omega_{2}, \omega_{2},-\omega_{1}\right) e^{j\left[2 \phi_{2}-\phi_{1}\right]} \\
& +\frac{50 A^{5}}{32} H_{5}\left(\omega_{2}, \omega_{2},-\omega_{1}, \omega,-\omega\right) e^{j\left[2 \phi_{2}-\phi_{1}\right]} \\
& +L S\left(A, 2 \omega_{2}-\omega_{1}\right)
\end{aligned}
$$

where $L S\left(A, 2 \omega_{2}-\omega_{1}\right)$ is the hereafter referred to large-signal contribution

$$
\begin{aligned}
& L S\left(A, 2 \omega_{2}-\omega_{1}\right) \\
& \quad \equiv A * \operatorname{TSDF}_{1}\left(A, 2 \omega_{2}-\omega_{1}\right)-\operatorname{SS}_{I_{\text {out }}}\left(2 \omega_{2}-\omega_{1}\right)
\end{aligned}
$$

meaning that $L S\left(A, 2 \omega_{2}-\omega_{1}\right)$ is near zero compared to the VS output, when the signal travels between $\mathbf{A}$ and $\mathbf{B}$ (if the circuit is biased between $\mathbf{A}$ and $\mathbf{B}$ ) and is dominant when the signal travels far from $\mathbf{B}$ toward $\mathbf{C}$.

In a memoryless system such as the one under consideration, if $I_{\text {out }}\left(2 \omega_{2}-\omega_{1}\right)$ has a phase of $0^{\circ}$ in its small-signal region (fundamental output power is again assumed as the reference phase), and then tends to $180^{\circ}$ for its large signal regime, it must have a zero, which results from the interaction between $\operatorname{LS}\left(A, 2 \omega_{2}-\omega_{1}\right)$ and the small-signal contribution $\mathrm{SS}_{I_{\text {out }}}\left(2 \omega_{2}-\omega_{1}\right)$.

In Section IV, some illustrative examples will be proposed in order to prove the applicability to real devices and systems.

\section{MESFET CASE STUdY}

In order to get a practical perspective of the proposed theory, let us imagine a simple circuit where a MESFET is connected to a resistive load $R_{L}$, as is shown in Fig. 3 . 
If the device's $I_{\mathrm{ds}}\left[V_{\mathrm{gs}}, V_{\mathrm{ds}}\right]$ nonlinear model is simultaneously solved with the appropriate boundary conditions imposed at its input and output

$$
\begin{aligned}
I_{\mathrm{ds}}(t) & =f_{N L}\left[V_{\mathrm{gs}}(t), V_{\mathrm{ds}}(t)\right]=I_{\mathrm{out}}(t) \\
V_{\mathrm{gs}}(t) & =V_{G G}+V_{s}(t)=V_{\mathrm{in}}(t) \\
V_{\mathrm{ds}}(t) & =V_{D D}-R_{L} \cdot I_{\mathrm{ds}}(t)
\end{aligned}
$$

(where $V_{s}(t)$ is the input voltage excitation and $V_{G G}$ and $V_{D D}$ are the gate and drain supply voltages, respectively), $I_{\text {out }}(t)$ can be represented as a direct transfer function of $V_{\text {in }}(t)$, which assumes a graphical aspect close to that of Fig. 2.

For the $I_{\mathrm{ds}}\left(V_{\mathrm{gs}}, V_{\mathrm{ds}}\right)$ nonlinearity, the Pedro's Model [8] was used due to its recognized ability on handling IMD simulation problems.

The above set of simultaneous equations was solved with an harmonic balance algorithm, which allowed the extraction of both the amplifier characteristic function and its first five derivatives, as required for the small-signal Volterra analysis of (1).

Note that neither the function $I_{\text {out }}\left(V_{\text {in }}\right)$ nor its derivatives $G, G_{3}, G_{5}$ (as represented in Fig. 4) have to be equal to the ones of $I_{\mathrm{ds}}\left(V_{\mathrm{gs}}, V_{\mathrm{ds}}\right)$ [5], $G_{m}, G_{m 3}, G_{m 5}$, except at bias points not far from pinchoff, where the influence of $V_{\mathrm{ds}}$ on $I_{\mathrm{ds}}\left(V_{\mathrm{in}}\right)$ is negligible. In fact, these derivatives are now defined by the Taylor expansion of $I_{\text {out }}\left(V_{\text {in }}\right)$

$$
\begin{aligned}
I_{\text {out }}\left(v_{\text {in }}(t)\right)=I_{\text {outDC }}+G v_{\text {in }}(t) & +G_{2} v_{\text {in }}(t)^{2}+G_{3} v_{\text {in }}(t)^{3} \\
& +G_{4} v_{\text {in }}(t)^{4}+G_{5} v_{\text {in }}(t)^{5} \cdots
\end{aligned}
$$

and can be calculated from

$$
\begin{aligned}
& h_{1}=G=\left.\frac{\partial f_{N L}\left(V_{\mathrm{in}}(t)\right)}{\partial V_{\mathrm{in}}(t)}\right|_{V_{\mathrm{in}}(t)=V_{\mathrm{in}} \mathrm{DC}} \\
& h_{2}=G_{2}=\left.\frac{1}{2 !} \frac{\partial^{2} f_{N L}\left(V_{\mathrm{in}}(t)\right)}{\partial^{2} V_{\mathrm{in}}(t)}\right|_{V_{\mathrm{in}}(t)=V_{\mathrm{in}} \mathrm{DC}} \\
& h_{3}=G_{3}=\left.\frac{1}{3 !} \frac{\partial^{3} f_{N L}\left(V_{\mathrm{in}}(t)\right)}{\partial^{3} V_{\mathrm{in}}(t)}\right|_{V_{\mathrm{in}}(t)=V_{\mathrm{in}} \mathrm{DC}} \\
& h_{4}=G_{4}=\left.\frac{1}{4 !} \frac{\partial^{4} f_{N L}\left(V_{\mathrm{in}}(t)\right)}{\partial^{4} V_{\mathrm{in}}(t)}\right|_{V_{\mathrm{in}}(t)=V_{\mathrm{in} \mathrm{DC}}} \ldots . \\
& h_{5}=G_{5}=\left.\frac{1}{5 !} \frac{\partial^{5} f_{N L}\left(V_{\mathrm{in}}(t)\right)}{\partial^{5} V_{\mathrm{in}}(t)}\right|_{V_{\mathrm{in}}(t)=V_{\mathrm{in}} \mathrm{DC}} \cdots
\end{aligned}
$$

The IMD at frequency $2 \omega_{2}-\omega_{1}$, including components up to fifth order, will be

$$
\mathrm{SS}_{I_{\text {out }}}\left(2 \omega_{2}-\omega_{1}\right)=\frac{3 A^{3}}{8} G_{3}+\frac{50 A^{5}}{32} G_{5} .
$$

Expression (7) states a strong relationship between the Volterra operators and $I_{\text {out }}\left(V_{\text {in }}\right)$ derivatives [6]. Thus, an observation of Fig. 4 leads to the conclusion that, as $G_{3}$ and $G_{5}$ change with the bias point, a tight control over small-signal IMD behavior can be gained by simply changing the device's

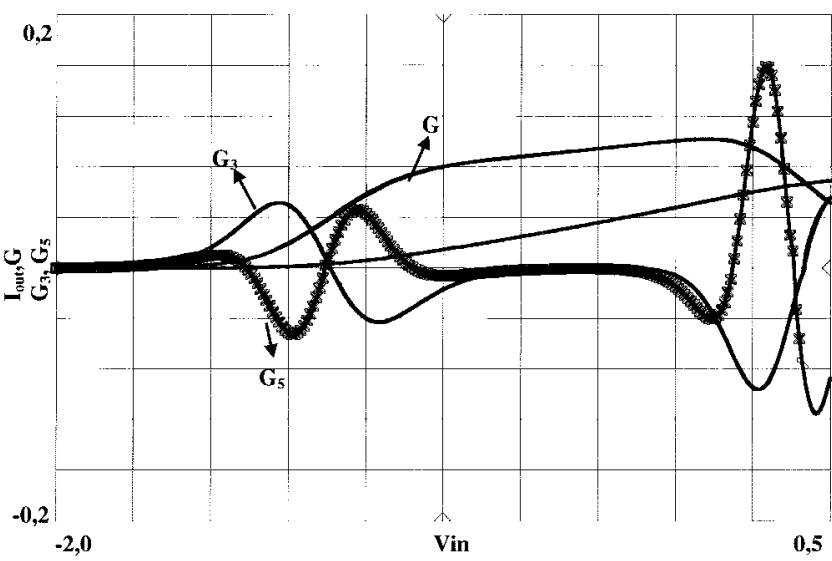

Fig. 4. Characteristic function: first, third, and fifth derivatives.

bias point. Therefore, as asymptotic large-signal IMD presents a $180^{\circ}$ phase, a distortion sweet spot can be produced by the careful selection of a quiescent point that has an associated $0^{\circ}$ phase small-signal IMD. On the other hand, if the bias point selection results on a $180^{\circ}$ small-signal IMD, the IMD versus drive pattern will not present any null, but a rapid increase on distortion, close to the 1-dB compression point.

In order to illustrate these theoretical assumptions, twotone harmonic balance simulations were performed on our nonlinear MESFET circuit for several bias conditions. Those simulation results of output power and IMD at $\omega_{1}$ and at $2 \omega_{2}-\omega_{1}$, respectively, are presented in Fig. 5 .

Case A corresponds to the device biased near pinchoff, a situation usually treated as a Class-B power amplifier $\left(V_{G G}=\right.$ $\left.V_{\text {inDC }}=-1.27 \mathrm{~V}\right)$. There, first the IMD rises at $3 \mathrm{~dB} / \mathrm{dB}$ for small-signal regimes and, when the output power starts to compress, an IMD minimum appears. The explanation for this pattern is that, at the referred bias, the small-signal IMD has the required $0^{\circ}$ phase, imposed by a positive $G_{3}$, while the large-signal IMD presents its asymptotic $180^{\circ}$.

In a practical circuit, the large-signal distortion has its origins on the device's strong nonlinearities, which, for a MESFET, can be either the triode to saturation zone transition or the input gate-channel diode conduction or breakdown. The one actually determining large-signal IMD mainly depends on which will be reached first as $V_{\mathrm{gs}}(t)$ and $V_{\mathrm{ds}}(t)$ control voltages increase. This, in turn, can be a priori predicted from the device's $I / V$ curves and the quiescent point considered for each particular design. Due to the moderate $V_{D D}$ voltages considered in the circuits studied in this paper, only the first two strong nonlinearities had nonnegligible impact.

In Case $\mathrm{B}$, the amplifier was biased for Class-AB operation with $V_{\mathrm{inDC}}=-0.97 \mathrm{~V}$. There, due to the negative $G_{3}$, the small-signal IMD has $180^{\circ}$ phase. As theoretically predicted in Section III, no IMD minimum is observed at the largesignal interface. Even so, and because at $V_{\mathrm{inDC}}=-0.97 \mathrm{~V}$ $G_{3}$ and $G_{\check{2}}$ have opposite signs, IMD compression appears near $P_{\text {in }}=0 \mathrm{dBm}$, before the sudden increase faced when signal level approaches large-signal excitation.

Case $\mathrm{C}$ is a Class-A power amplifier, biased at $V_{\mathrm{inDC}}=$ $-0.45 \mathrm{~V}$, where $G_{3}$ is again negative, but now with the same sign as $G_{\tilde{5}}$. In this situation, the IMD curve expands 


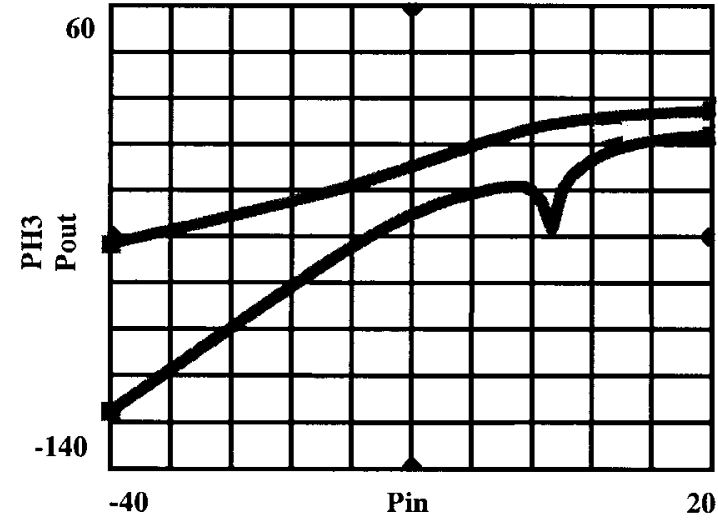

(a)

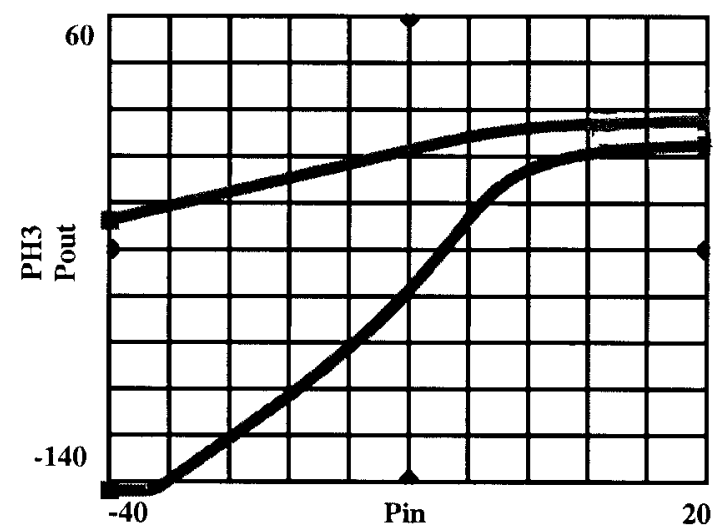

(c)

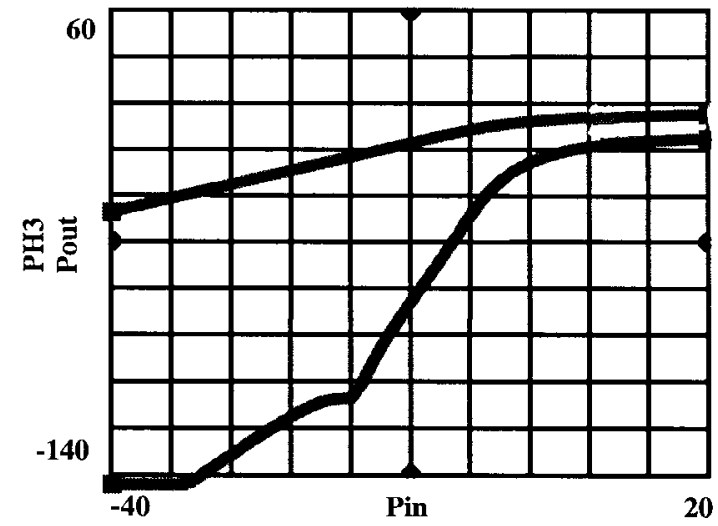

(e)

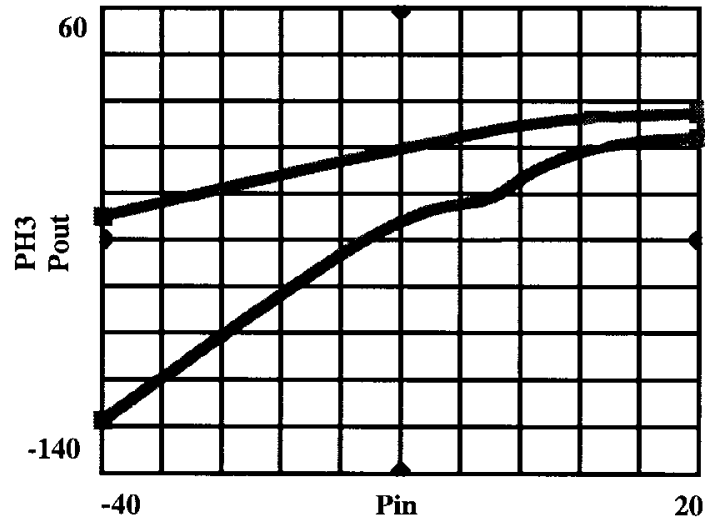

(b)

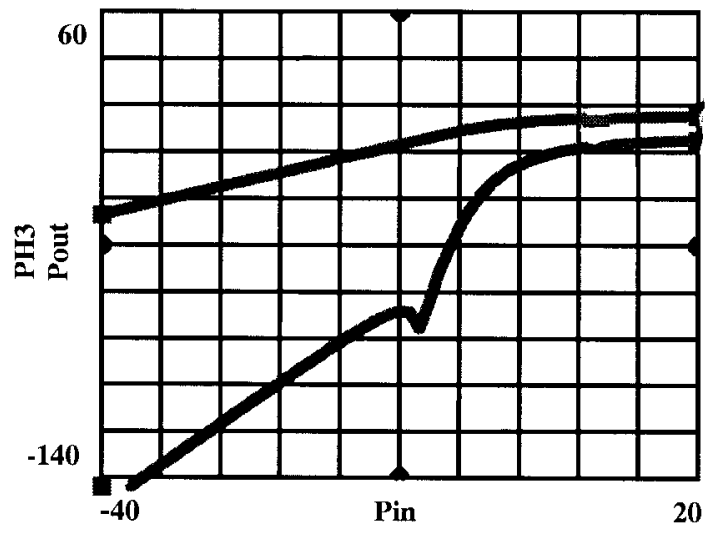

(d)

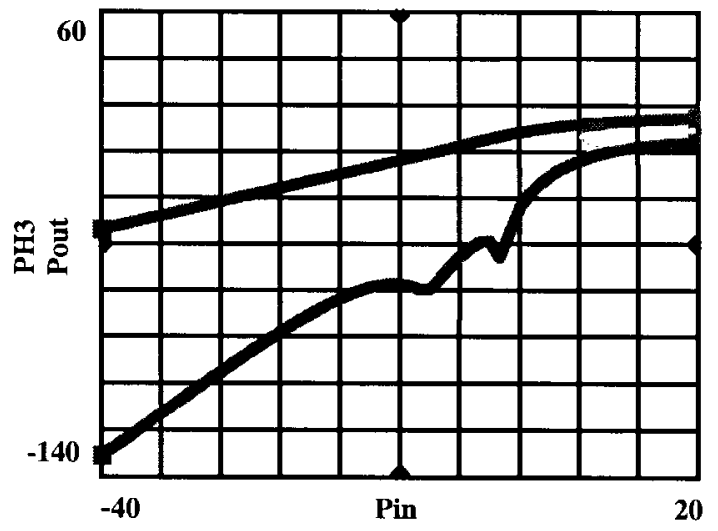

(f)

Fig. 5. Output power and IMD for: (a) Class B. (b) Class AB. (c) Class A(-). (d) Class A (+). (e) Small-signal sweet spot. (f) Double minimum.

at small signal and compresses at large signal. As predicted, no minimum is evident.

In Case $\mathrm{D}$, the device was again biased for Class $\mathrm{A}$, $V_{\mathrm{inDC}}=-0.31 \mathrm{~V}$, but now with a greater quiescent current. $G_{3}$ is now positive and an IMD minimum appears at the interaction between small- and large-signal IMD, as was the case already verified for Class-B operation.

In Case $\mathrm{E}$, the device was biased at its small-signal sweet spot [7], $V_{\mathrm{inDC}}=-0.4 \mathrm{~V}$, where $G_{3} \approx 0$, and $G_{\tilde{5}}$ is predominant. Despite the IMD, curve power can still be explained in the same manner, its small-signal power is now controlled by $G_{5}$, as is clear from the observed low distortion power, and its distinct $5-\mathrm{dB} / \mathrm{dB}$ slope.
Finally, for completeness, in Case $\mathrm{F}$, the device was biased in a point where $G_{3}<0, G_{5}>0$ and $G_{3} \approx G_{5}: V_{\mathrm{inDC}}=$ $-1.1 \mathrm{~V}$. In that case, a double minimum is evident. The first minimum is caused by the interaction between the small-signal third- and fifth-order components imposed by the selected values of $G_{3}$ and $G_{\tilde{5}}$, while the second again has its origins on the small- (now controlled by $G_{5}$ ) and large-signal IMD components' cancellation. Indeed, $G_{5}>0$, and now represents the dominant contribution to small-signal IMD, just before the large-signal interface.

All of the above conclusions regarding the bias point IMD dependence were developed from a very simplified memoryless circuit. In what follows, restriction will be lift 


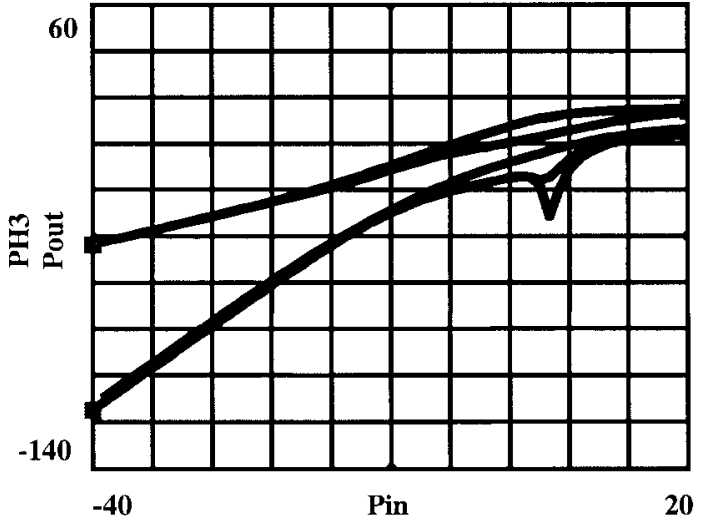

(a)

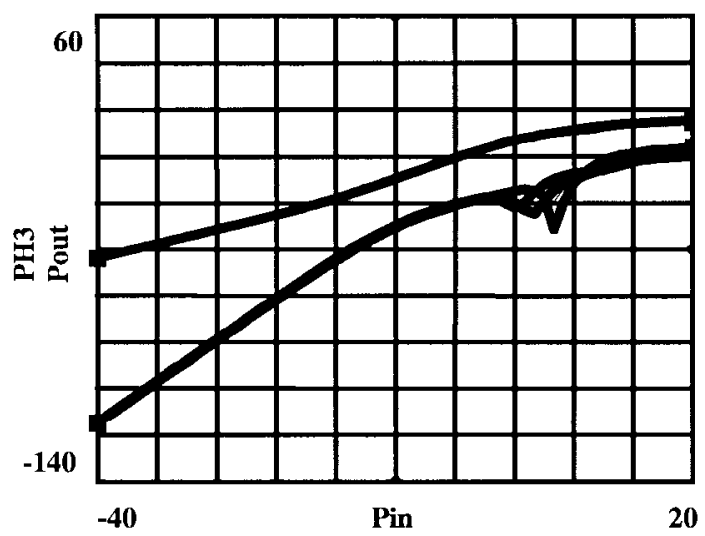

(c)

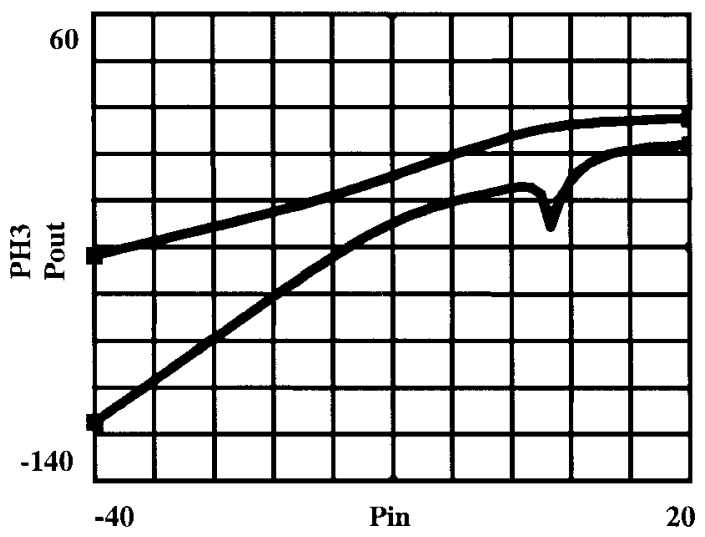

(e)

Fig. 6. Output power and IMD for each matching network load pulling: upper harmonics.

off in order to study the variation in amplifier IMD pattern induced by changes in its terminating matching networks.

Since the amplifier now has memory, the simplified Taylor series expansion turns into a full VS analysis. Since such a VS expansion was already previously developed by many authors [3], [5]-[7], it will not be fully discussed here, and we will only retain the part that we consider more important for our explanatory purposes.

For illustration, let the device be biased in a quiescent point near or beyond pinchoff. Due to the very small gains associated to such bias points, it can be shown that there, only certain coefficients of the Taylor series expansion of

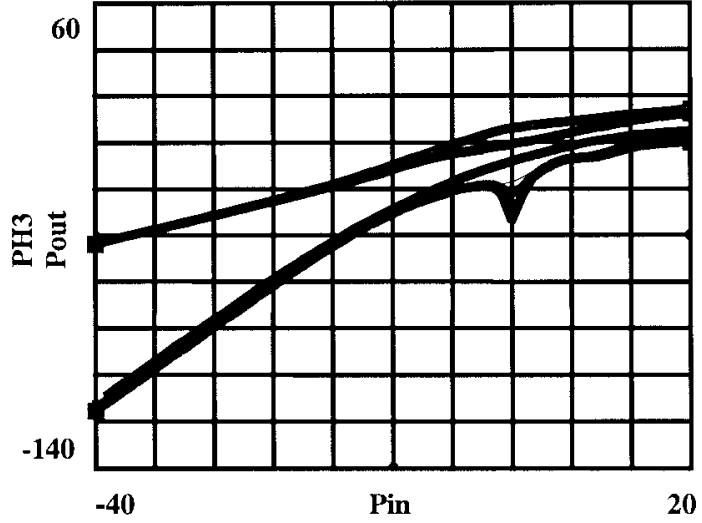

(b)

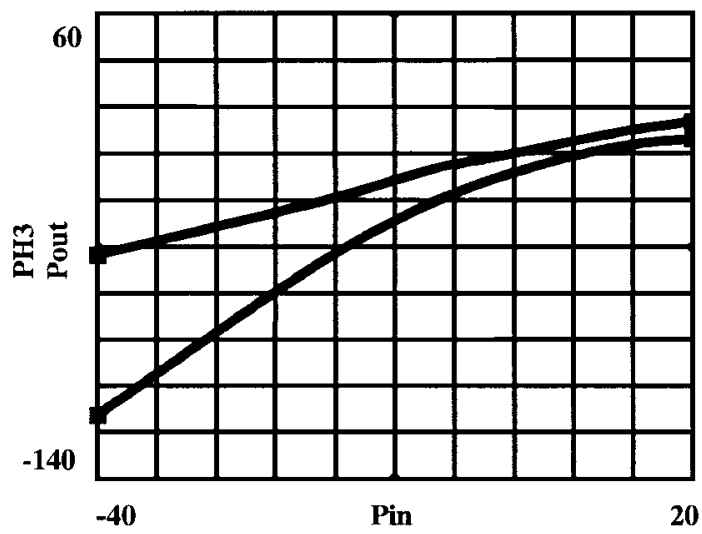

(d)

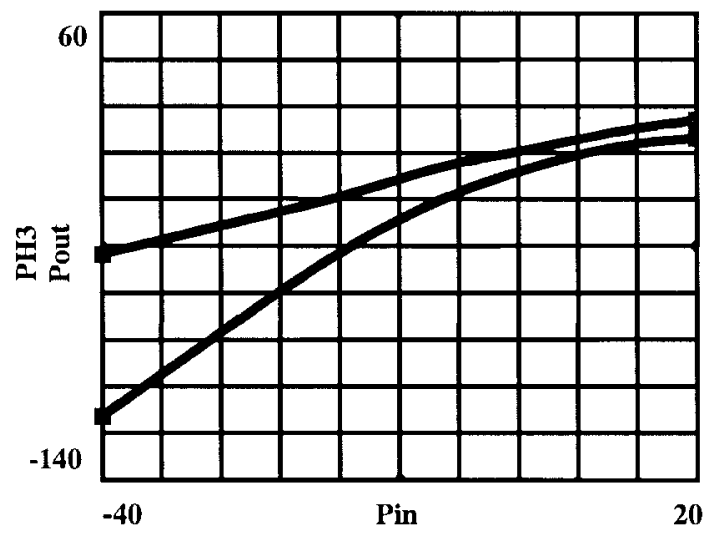

(f)

(a)-(b) Baseband. (c)-(d) Second harmonic. (e)-(f) Third, fourth, fifth, and

$$
I_{\mathrm{ds}}\left(V_{\mathrm{gs}}, V_{\mathrm{ds}}\right)
$$

$$
\begin{aligned}
G_{m} & =\frac{\partial I_{\mathrm{ds}}}{\partial V_{\mathrm{gs}}} \\
G_{m 2} & =\frac{1}{2 !} \frac{\partial^{2} I_{\mathrm{ds}}}{\partial 2 V_{\mathrm{gs}}} \\
G_{m 3} & =\frac{1}{3 !} \frac{\partial^{3} I_{\mathrm{ds}}}{\partial^{3} V_{\mathrm{gs}}} \\
G_{\mathrm{ds}} & =\frac{\partial I_{\mathrm{ds}}}{\partial V_{\mathrm{ds}}} \\
G_{m d} & =\frac{1}{2 !} \frac{\partial I_{\mathrm{ds}}}{\partial V_{\mathrm{gs}} \partial V_{\mathrm{ds}}}
\end{aligned}
$$


TABLE I

Different Harmonic Matching Network Cases

\begin{tabular}{|c|c|c|c|}
\hline Arrangement & $S_{\text {load }}$ at Base-Band & $S_{\text {load }}$ at $2^{\text {nd }}$ Harm. & $S_{\text {load }}$ at $3^{\text {rd }}$ and upper \\
\hline $\mathrm{A}$ & Variable & $1\left\lfloor 180^{\circ}\right.$ & $1180^{\circ}$ \\
\hline $\mathrm{B}$ & Variable & $1 \mid \underline{0}^{\circ}$ & $1180^{\circ}$ \\
\hline $\mathrm{C}$ & $1\left[180^{\circ}\right.$ & Variable & $1180^{\circ}$ \\
\hline $\mathrm{D}$ & $1 \underline{0^{\circ}}$ & Variable & $1 \mid 180^{\circ}$ \\
\hline $\mathrm{E}$ & $1180^{\circ}$ & $1180^{\circ}$ & Variable \\
\hline $\mathrm{F}$ & $1{\underline{0^{\circ}}}^{-}$ & $1180^{\circ}$ & Variable \\
\hline
\end{tabular}

are important and, thus, the Volterra nonlinear operators will be

$$
\begin{aligned}
H_{1}(\omega) & =\frac{G_{m}}{1+G_{\mathrm{ds}} Z_{L}(\omega)} \\
H_{2}\left(\omega_{1}, \omega_{2}\right) & =\frac{2 G_{m 2}+G_{m d} H_{1}\left(\omega_{1}\right)+G_{m d} H_{1}\left(\omega_{2}\right)}{2+2 G_{\mathrm{ds}} Z_{L}\left(\omega_{1}+\omega_{2}\right)}
\end{aligned}
$$

and

$$
\begin{aligned}
H_{3}\left(\omega_{1}, \omega_{2}, \omega_{3}\right)= & \frac{1}{6+6 G_{\mathrm{ds}} Z_{L}\left(\omega_{1}+\omega_{3}+\omega_{3}\right)} \\
& \cdot\left(6 G_{m 3}+2 G_{m d} Z_{L}\left(\omega_{1}+\omega_{2}\right) H_{2}\left(\omega_{1}, \omega_{2}\right)\right. \\
& +2 G_{m d} Z_{L}\left(\omega_{1}+\omega_{2}\right) H_{2}\left(\omega_{1}, \omega_{3}\right) \\
& \left.+2 G_{m d} Z_{L}\left(\omega_{2}+\omega_{3}\right) H_{2}\left(\omega_{3}, \omega_{2}\right)\right) . \quad(11)
\end{aligned}
$$

As can be seen from the above expressions, if we consider the two-tone IMD at $2 \omega_{2}-\omega_{1}, I_{\mathrm{ds}}\left(2 \omega_{2}-\omega_{1}\right)=3 / 8 H_{3}\left(2 \omega_{2}-\right.$ $\left.\omega_{1}\right) * A^{3}$, where

$$
\begin{aligned}
H_{3}\left(2 \omega_{1}-\omega_{1}\right)= & \frac{1}{6+}+6 G_{\mathrm{ds}} Z_{L}\left(2 \omega_{2}-\omega_{1}\right) \\
& \cdot\left(6 G_{m 3}+2 G_{m d} Z_{L}\left(2 \omega_{2}\right) H_{2}\left(\omega_{2}, \omega_{2}\right)\right. \\
& \left.\quad+4 G_{m d} Z_{L}\left(\omega_{2}-\omega_{1}\right) H_{2}\left(\omega_{2},-\omega_{1}\right)\right)
\end{aligned}
$$

with

$$
\begin{aligned}
H_{2}\left(\omega_{2}, \omega_{2}\right) & =\frac{2 G_{m 2}+2 G_{m d} H_{1}\left(\omega_{2}\right)}{2+2 G_{\mathrm{ds}} Z_{L}\left(2 \omega_{2}\right)} \\
H_{2}\left(\omega_{2},-\omega_{1}\right) & =\frac{2 G_{m 2}+G_{m d} H_{1}\left(\omega_{2}\right)+G_{m d} H_{1}\left(-\omega_{1}\right)}{2+2 G_{\mathrm{ds}} Z_{L}\left(\omega_{2}-\omega_{1}\right)} .
\end{aligned}
$$

Then, considering that $Z_{L}\left(\omega_{2}-\omega_{1}\right)$ (baseband load impedance presented by the output matching network) and $Z_{L}\left(2 \omega_{2}\right)$ (similar second harmonic load impedance) are varied, it is possible to modify the phase of the small-signal behavior of the amplifier $H_{3}\left(2 \omega_{2}-\omega_{1}\right)$. This, in turn, may induce a change in the IMD pattern, compared to the one previously observed in Fig. 5(a).

To test this hypothesis, an independent load-pull simulation at the baseband and second harmonic was performed again with a harmonic balance machine (load impedances at the fundamental frequencies were maintained constant and equal to $50 \Omega$ ). Fig. 6 and Table I summarize those results. A "variable" statement for any load reflection coefficient means that the four reactive values were tried, i.e., $S_{\text {load }}=1\left\lfloor\theta^{\circ}\right.$ where $\theta=0^{\circ}, 90^{\circ}, 180^{\circ}$, and $270^{\circ}$.

As is clear from Cases A and B, changes in the baseband matching network can cause the elimination of the IMD

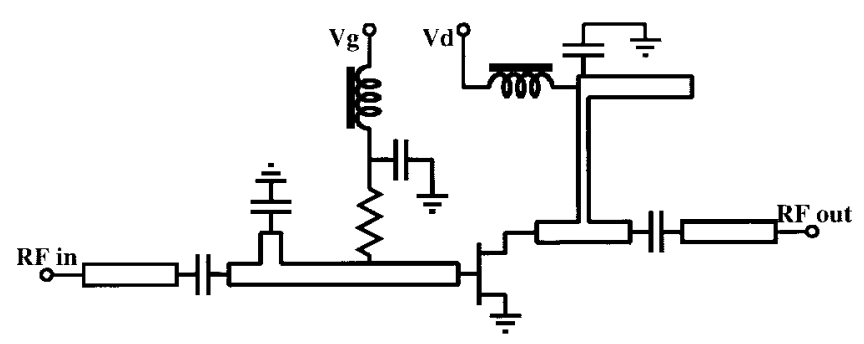

Fig. 7. MESFET power-amplifier prototype used for laboratory validation.

minimum (for an open-circuit baseband load). This can be explained by considering (12), where a change in $Z_{\mathrm{Load}}\left(\omega_{2}-\right.$ $\omega_{1}$ ) may reverse the phase of the small-signal IMD and, thus, the IMD minimum can no longer be present.

In Cases $\mathrm{C}$ and $\mathrm{D}$, the second harmonic was tuned. The load-pull simulation results now indicate that, for a shortcircuit baseband load, the variation in the second harmonic loading changes the IMD pattern, as was predictable from (12). However, if the baseband load is an open circuit, the second harmonic tuning has no effect on the IMD pattern, which is a consequence of the stronger impact of the baseband load on IMD than the one of second harmonic. This, again, could be also derived from a careful observation of (12).

In the final Cases $\mathrm{E}$ and $\mathrm{F}$, the matching networks greater than the second harmonic were varied. There, since smallsignal IMD cannot be affected, as is predicted by (12), and large-signal IMD remains essentially unaltered, no change in IMD could be perceived.

In conclusion, it can be said that if the goal is an IMD minimum, then the optimum output-matching network should present a very low impedance to both the baseband and second harmonic. In fact, generally, only the second harmonic load has to be controlled, as the baseband short circuit can be easily guaranteed by a wise drain bias circuitry.

\section{APPLICATION EXAMPLE}

In order to prove the practical validity of the proposed theory, a single-stage $C$-band MESFET power amplifier was designed for maximum output power and optimum IMD pattern (see Fig. 7). That is, the input matching network was tuned for maximum gain while, at the output, optimum Cripps load for the fundamental and short-circuit for the baseband and second harmonic loads were used. The model used for the MESFET device is equivalent to the previously presented in Figs. 3 and 4 , with a $V_{T}=-1.25 \mathrm{~V}$.

In this application example, two different bias points were used, one for Class-AB operation $\left(V_{\mathrm{gs}_{s}}=-0.61 \mathrm{~V}\right)$, and 


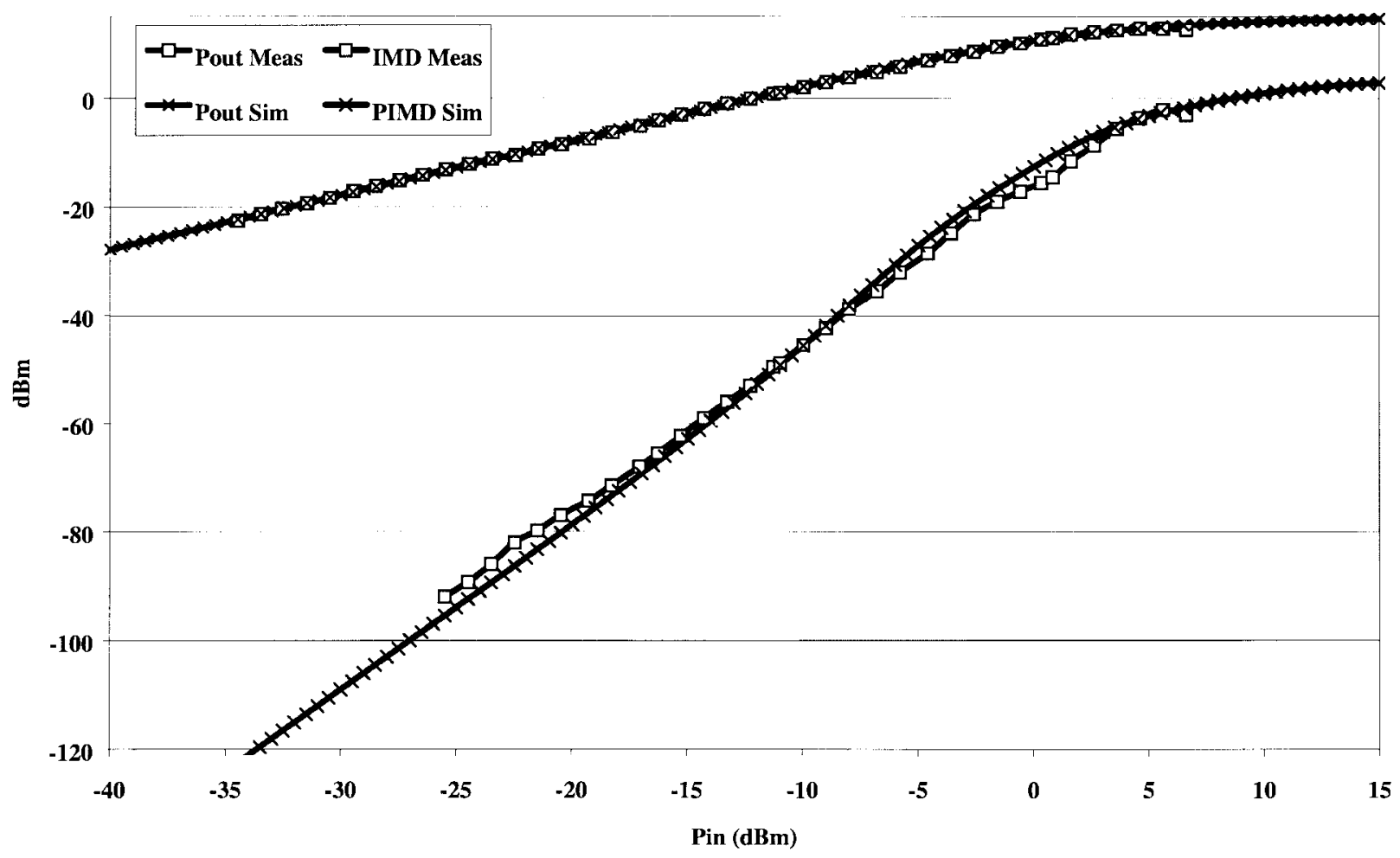

Fig. 8. Amplifier $P_{\text {in }}-P_{\text {out }}$ fundamental and IMD curves (measured and simulated) for Class-AB operation $\left(V_{\text {in }}=-0.61 \mathrm{~V}\right)$.

another for Class-B $\left(V_{\mathrm{gs}}=-1.24 \mathrm{~V}\right)$. In both of these situations, the amplifier was excited by two-tone and narrowband Gaussian noise signals. The corresponding measured and simulated results were then compared, taking into account the predictions inferred from the above explained theory. The simulator used for the noise signal is a state-ofthe-art in-house-developed harmonic balance machine [9], [10].

\section{A. Two-Tone Case}

Fig. 8 shows measured and simulated results of fundamental $P_{\text {out }}\left(\omega_{2}=2 \pi * 2.001 \mathrm{GHz}\right)$ and in-band two-tone IMD $\left(2 \omega_{2}-\omega_{1}=2 \pi * 2.003 \mathrm{GHz}\right)$ versus $P_{\mathrm{in}}$ curves. These results were obtained when the device was biased for Class AB. They represent the typical gain compression behavior and associated monotonic IMD rise with input drive level.

Now consider that the gate bias of this circuit is changed in order to have a positive $G_{3},\left(V_{\text {in }}=-1.24 \mathrm{~V}\right)$. The transistor would then be biased near Class B. Using (5), we can predict that a large-signal sweet spot would appear when the input signal excursion travels far beyond point $\mathbf{B}$ (Fig. 2) since there $L S\left(A, 2 \omega_{2}-\omega_{1}\right)$ would rise and tend to an opposite phase in-band IMD.

The measured and simulated fundamental and in-band IMD output powers versus drive for this amplifier can be observed in Fig. 9. The large-signal IMD sweet spot is clearly evident near the 1-dB compression point. Thus, by only changing the bias point, it was possible to reduce the IMD power near the 1-dB compression point by over $20 \mathrm{~dB}$.

Fig. 10 represents intermodulation ratio (IMR) versus output power for these Class-AB and Class-B amplifiers in order to il- lustrate the usefulness of the proposed theoretical explanation.

It is possible to see that for an equivalent output power close to $10 \mathrm{dBm}$, the IMR is better for the Class-B power amplifier than for the Class-AB design. Despite the better small-signal IMR presented by Class $A B$, it is still possible to generate a better IMR with a Class-B amplifier for the same output transmitted power located on the onset of saturation.

In summary, for a certain IMR specification it is possible to use a Class-B amplifier, taking all its recognized advantages in drain efficiency, in applications where traditional designs would advise the more obvious quasi-linear Class AB. Also, all these conclusions could be a priori predicted from simulations, as is guaranteed from the remarkable agreement obtained from the laboratory and computed results.

\section{B. Multitone Excitation (Narrow-Band Gaussian Noise)}

In order to prove the validity of the above model for telecommunications applications, a more realistic input spectrum (Fig. 11) was used. The input spectrum was built using a baseband Gaussian noise generator that was filtered to have a contained spectrum inside a precise bandwidth. This baseband signal was then used to modulate a $2-\mathrm{GHz}$ carrier (AM modulation). Output signal power, and adjacent channel power (ACP) ratio were measured and simulated on that microwave power amplifier for an input power sweep (Fig. 12). The two above-referred bias conditions were again considered.

Equal channel spacing and signal bandwidth of $600 \mathrm{kHz}$ were imposed, as is marked by the vertical arrows presented on the $x x$-axis of Fig. 11. ACP was herein defined as the ratio between integrated fundamental power and upper lobe spectral regrowth power. 


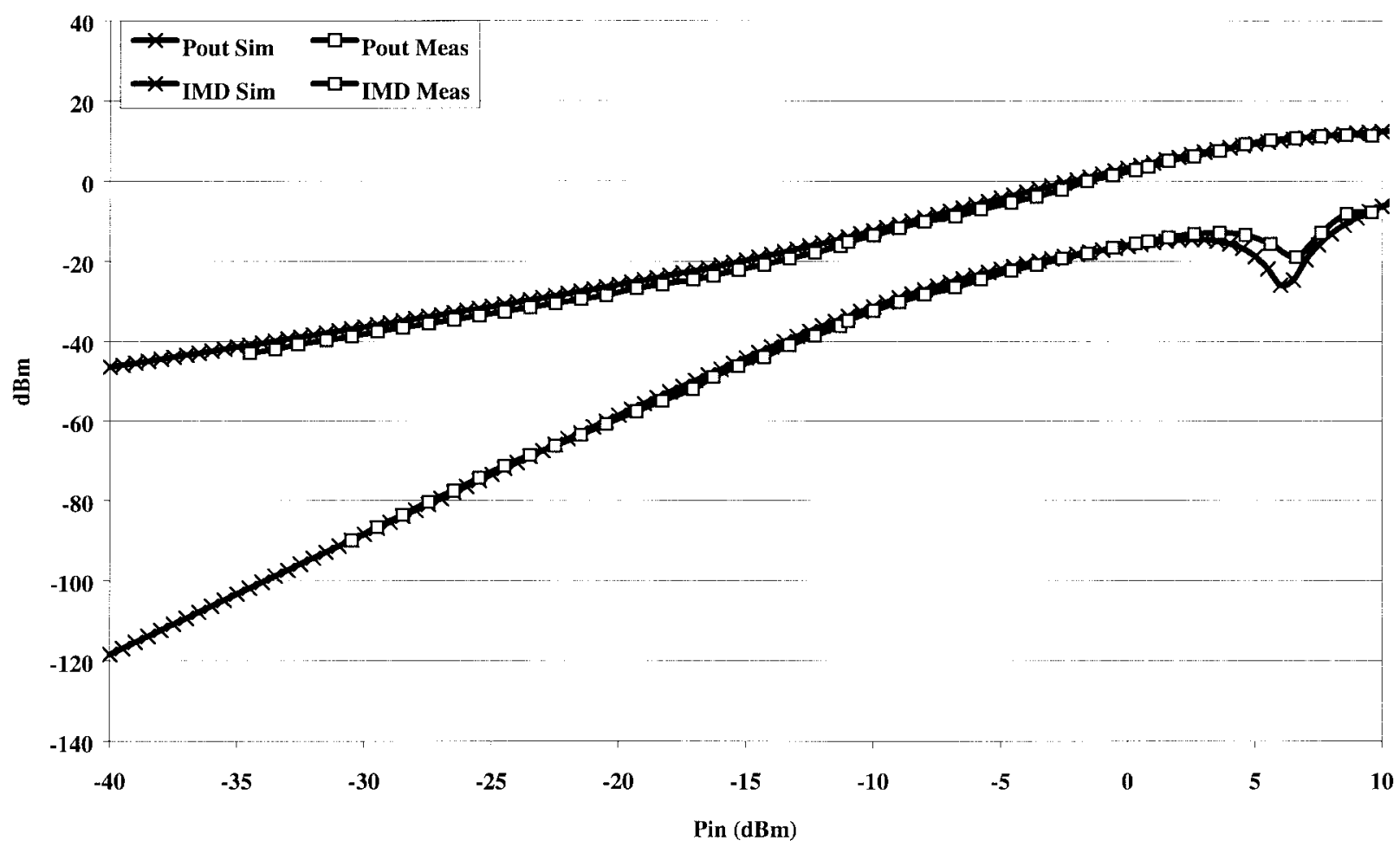

Fig. 9. Amplifier $P_{\text {in }}-P_{\text {out }}$ fundamental and IMD curves (measured and simulated) for Class-B operation $\left(V_{\text {in }}=-1.24 \mathrm{~V}\right)$.

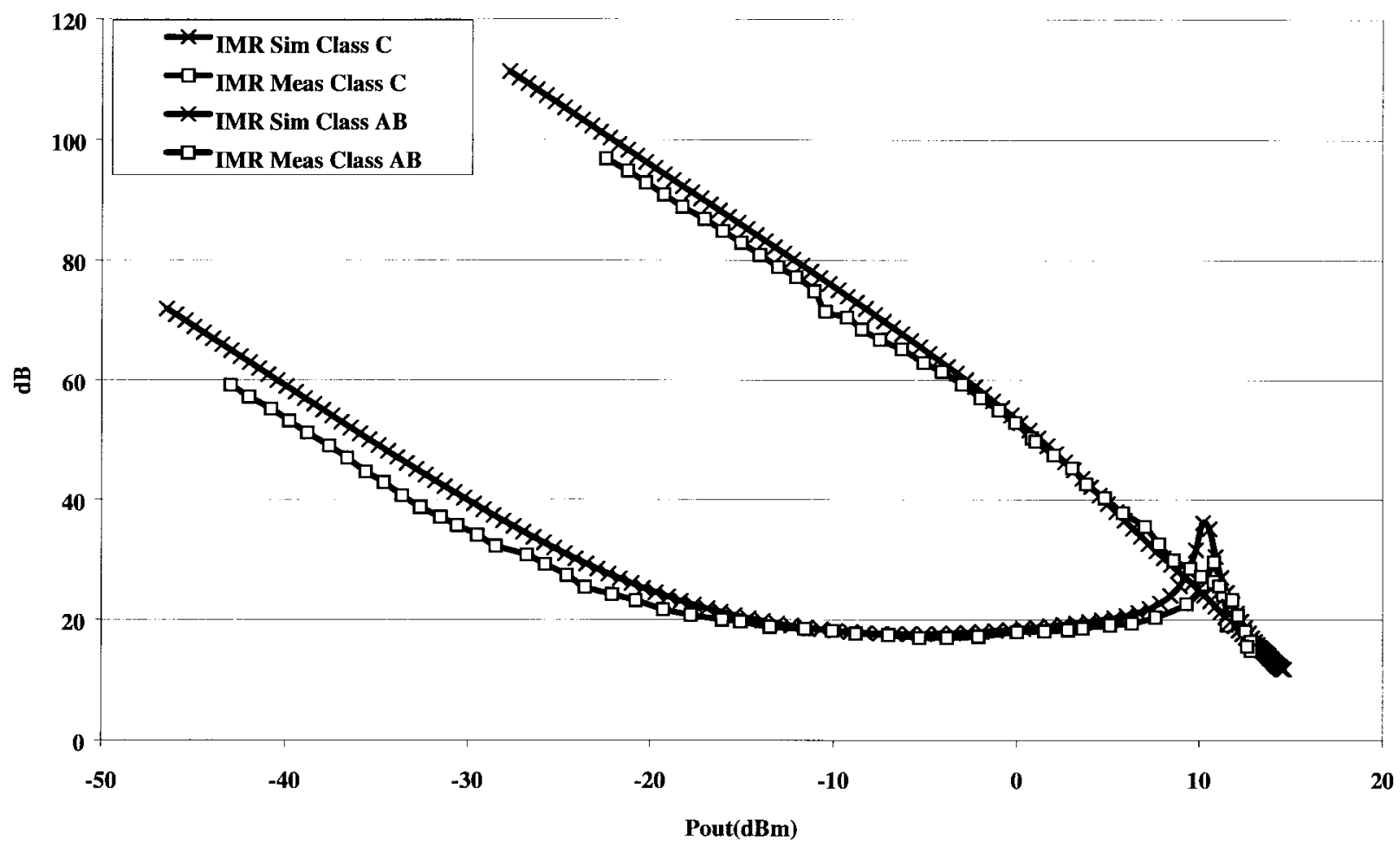

Fig. 10. IMR for two similar Class-AB and Class-B power amplifiers.

Looking at the experimental results of Figs. 8, 9, and 12, the first important conclusion to be drawn is that two-tone IMD and noise distortion (ACP) are very well correlated. In fact, the qualitative statements above referred for the twotone test maintain its applicability despite the increase in excitation complexity. Also, when comparing the performance gathered with the two quiescent points tested, over $10 \mathrm{~dB}$ of increase in observed ACP for the Class-B design can again be achieved near the 1-dB compression point for almost the same fundamental transmitted power. 


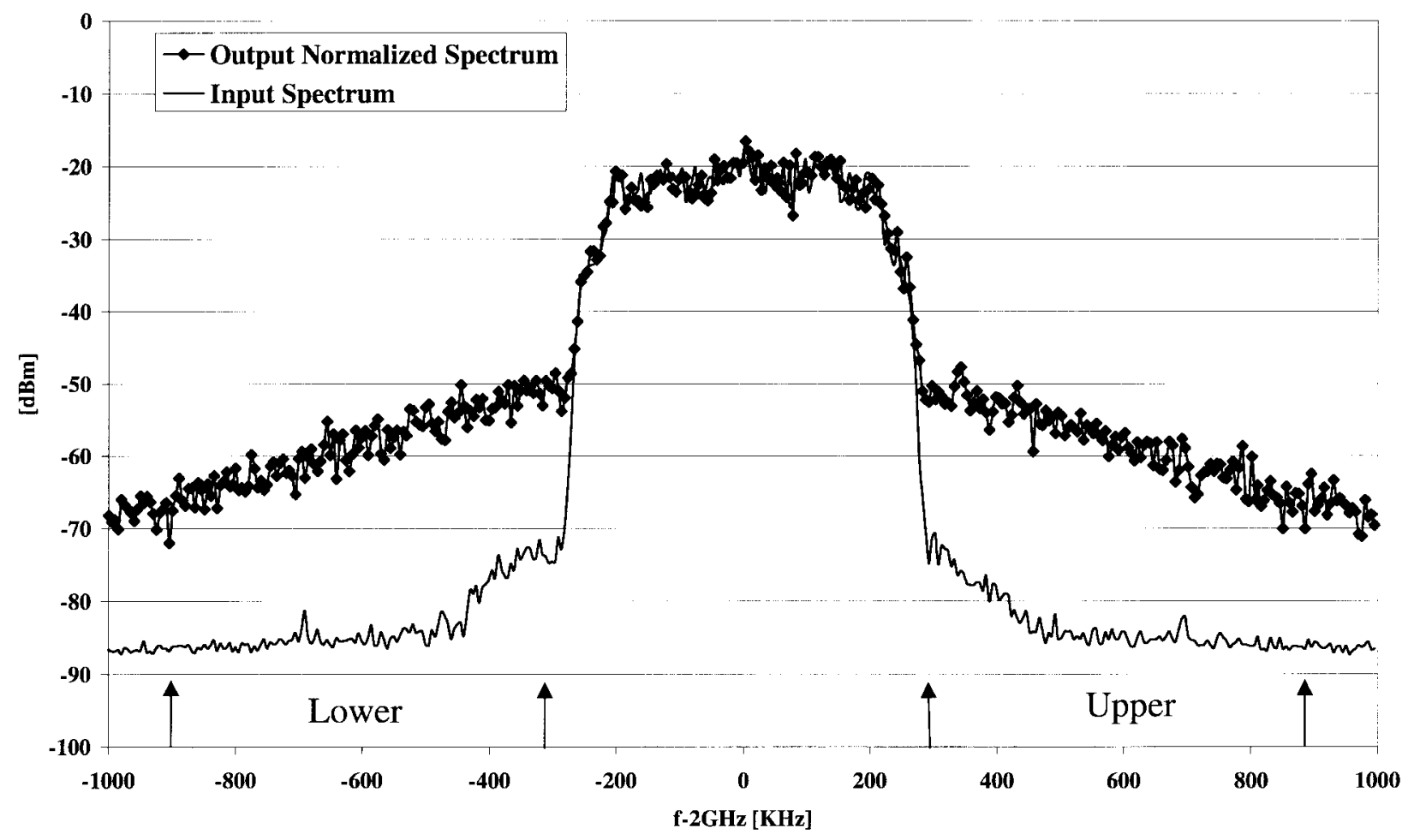

Fig. 11. Narrow-band Gaussian noise input and output distorted spectrum.

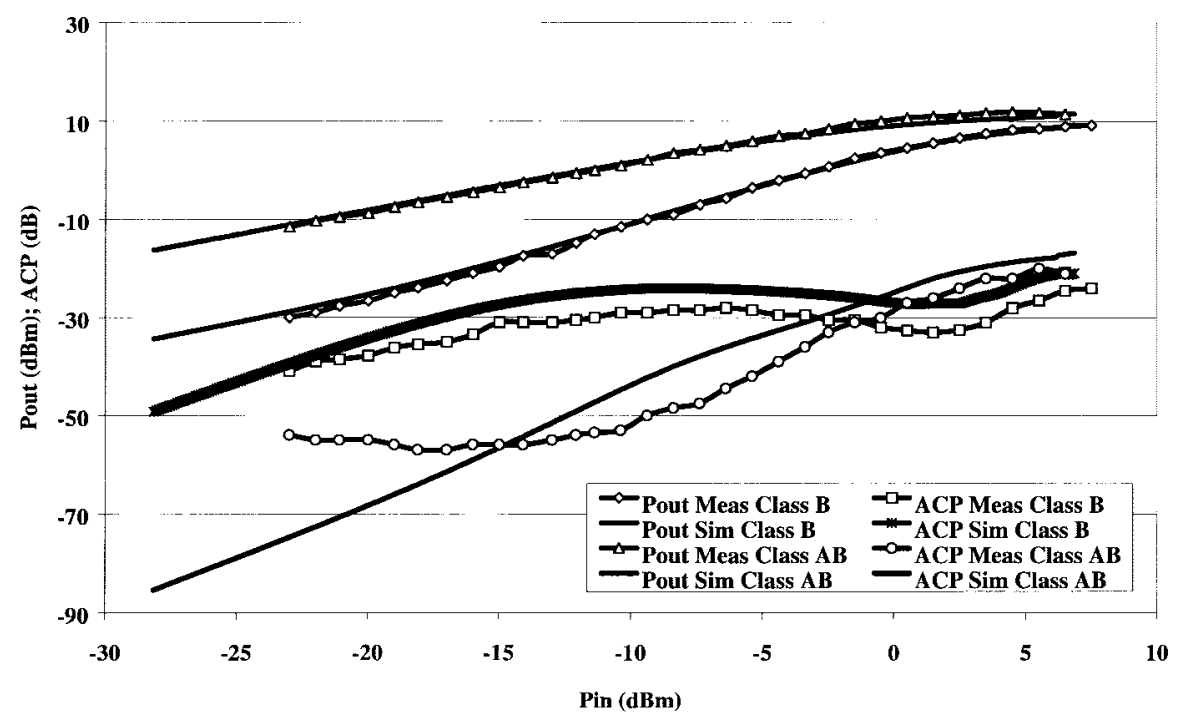

Fig. 12. Total measured output power and ACP for real-spectrum-excitation narrow-band Gaussian noise.

Let us now compare the simulations with the measurement results. A first glance indicates an almost perfect matching, as long as fundamental power is concerned.

In the case of ACP, the results are not so precise. The reasons for that are mainly related to the difficulty in accurately measuring the power associated with a randomly varying signal. First, any two successive ACP measurements could vary by as much as $\pm 2 \mathrm{~dB}$, which is within the average simulation results discrepancies. The large differences observed for the Class-AB small-signal regime are entirely due to the mask imposed by the measurement noise floor. The other differences observed in the small- and large-signal interfaces are attributed to imperfections in the nonlinear model used for the $I_{\mathrm{ds}}\left(V_{\mathrm{gs}}\right.$, $V_{\mathrm{ds}}$ ) nonlinearity. In fact, this nonlinear MESFET model was optimized to present a good fit up to the third-order device's nonlinearity, but it can not easily control the fifth [8]. Also, as was seen above, that is the ultimate responsible for the observed compression of small-signal IMD. Finally, since we are now (contrary to any simulation) dealing with continuous spectrum densities with finite slopes, it is much more difficult to define a precise border between the large fundamental output power and the weaker adjacent channel. In our case, these errors could be as high as $\pm 3 \mathrm{~dB}$. Nevertheless, the overall agreement is quite good, which proves the applicability of 
the proposed theory to integrate small- and large-signal IMD behavior, and guarantees that the large-signal IMD sweet spots can be predicted on an early power-amplifier design phase.

A final note on the presented measured and simulated results should refer to the fact that (and as far as the authors are aware) this is the first time that real nonlinear distortion measurement and simulated results for a noise excitation are compared. This proves that the used computation resources [8]-[10], although still in their infancy, already constitute valuable tools for complex nonlinear distortion prediction and evaluation.

\section{CONCLUSIONS}

In this paper, a new mathematical model was proposed to explain and predict the various IMD versus drive-level patterns normally encountered on usual RF and microwave power amplifiers. A simple approach was used for the design of power amplifiers specially tailored to present a desired IMD behavior. By varying the active device's bias point and/or its baseband/second harmonic load terminations, it was possible to improve the two-tone IMD or ACP near the 1-dB compression point by over $10 \mathrm{~dB}$. To our knowledge, this is the first time this type of large-signal IMD sweet spots was investigated and predicted using a mathematical model.

Finally, a special in-house developed harmonic balance machine enabled the extension to random signals of the theoretically predicted two-tone IMD sweet-spots conditions.

\section{REFERENCES}

[1] S. R. Novis and L. Pelletier, "IMD parameters describe LDMOS device performance," Microwaves RF, vol. 37, no. 7, pp. 69-74, July 1998.

[2] C. Blanco, "Gain expansion and intermodulation in a MESFET amplifier," Electron. Lett., vol. 15, no. 1, pp. 31-32, Jan. 1979.

[3] S. A. Maas, Nonlinear Microwave Circuits. Norwood, MA: Artech House, 1988

[4] A. Gelb and W. E. V. Velde, Multiple-Input Describing Functions and Nonlinear System Design. New York: McGraw-Hill, 1968.

[5] J. C. Pedro e J. Perez, "Accurate simulation of GaAs MESFET's intermodulation distortion using a new drain-source current model," IEEE Trans. Microwave Theory Tech., vol. 42, pp. 25-33, Jan. 1994.

[6] S. A. Maas, "How to model intermodulation distortion," in IEEE MTT-S Microwave Symp. Dig., Boston, MA, June 1991, pp. 149-151.
[7] J. C. Pedro, "Evaluation of MESFET nonlinear intermodulation distortion reduction by channel doping control," IEEE Trans. Microwave Theory Tech., vol. 45, pp. 1989-1997, Nov. 1997.

[8] J. C. Pedro and J. Perez, "A novel nonlinear GaAs FET model for intermodulation analysis in general purpose harmonic balance simulators," in Proc. 23rd European Microwave Conf., Madrid, Spain, Sept. 1993, pp. 714-716.

[9] N. B. Carvalho and J. C. Pedro, "Multitone frequency domain simulation of nonlinear circuits in large- and small-signal regimes," IEEE Trans. Microwave Theory Tech., vol. 46, pp. 2016-2024, Dec. 1998.

[10] J. C. Pedro and N. B. Carvalho, "Efficient harmonic balance computation of microwave circuits response to multi-tone spectra," presented at the 29th European Microwave Conf., Munich, Germany, Oct. 1999.

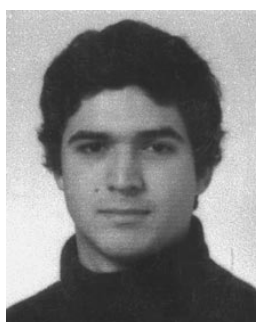

Nuno Borges de Carvalho (S'92) was born in Luanda, Portugal, in 1972. He received the diploma degree in electronics and telecommunications engineering from the Universidade de Aveiro, Aveiro, Portugal, in 1995, and is currently working toward the Ph.D. degree.

In 1997, he was appointed Assistant Lecturer at the Universidade de Aveiro. His main research interests include computer-aided design (CAD) for nonlinear circuits and active device modeling and design.

Mr. Carvalho is a member of the Portuguese Engineering Association. He was the recipient of the 1995 University of Aveiro and the Portuguese Engineering Association Prize for the best 1995 student at the Universidade de Aveiro, and the 1998 Student Paper Competition (third place) presented at the IEEE International Microwave Symposium. He was also a recipient of a Praxis XXI Grant.

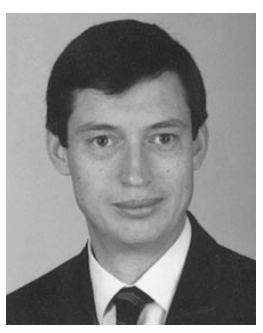

José Carlos Pedro (S'90-M'95) was born in Espinho, Portugal, on March 7, 1962. He received the diploma and doctoral degrees in electronics and telecommunications engineering, from the Universidade de Aveiro, Aveiro, Portugal, in 1985 and 1993 respectively.

From 1985 to 1994, he was an Assistant Lecturer at the Universidade de Aveiro, became a Professor in 1993, and is currently an Associate Professor. He is also a Senior Research Scientist at the Instituto de Telecomunicações, Universidade de Aveiro. His main scientific interests include active device modeling and the analysis and design of various nonlinear microwave and optoelectronics circuits, in particular, the design of highly linear multicarrier power amplifiers.

Dr. Pedro received the 1993 Marconi Young Scientist Award. 